\title{
The effect of two sphenoidotomy approaches on the sense of smell: trans-ethmoidal versus trans-sphenoethmoidal*
}

\author{
Andrew Thamboo, Brent A. Chang, Al-Rahim Habib, Jamil Manji, \\ lain Hathorn, Vishnu Sunkaraneni, Amin Javer
}

Division of Otolaryngology, University of British Columbia, St. Paul's Sinus Centre, Vancouver, British Columbia, Canada
Rhinology 52: 281-287, 2014 DOI:10.4193/Rhino13.106

*Received for publication:

July 22,2013

Accepted: January 31, 2014

\begin{abstract}
Background: There are generally two methods to access the sphenoid sinus: either through the natural ostium (trans-sphenoethmoidal or via sphenoethmoidal recess), or by creating a second opening through the posterior ethmoids (trans-ethmoidal). This study psychophysically and subjectively evaluates the effect of the trans-sphenoethmoidal technique to the trans-ethmoidal technique for sphenoid sinusotomy on olfactory function.
\end{abstract}

Methods: Prospective cohort analysis of 48 patients with comparable sinus disease underwent primary sphenoidotomy via transsphenoethmoidal $(n=24)$ versus trans-ethmiodal $(n=24)$ technique between September 2011 and February 2012. The patients had their olfaction measured psychophysically with "Sniffin' Sticks" and subjectively with a visual analogue scale (VAS) pre-operatively and at 5 weeks post-operatively.

Results: Psychophysical scores from the Sniffin' sticks provide a Threshold, Discrimination and Identification (TDI) score out of 48. The TDI change (post-operative TDI score minus pre-operative score) as well as VAS change (post-operative VAS minus pre-operative VAS) were analyzed using t-test analysis, which showed no significant difference between the two measurements.

Conclusion: If the trans-sphenoethmoidal technique is done meticulously, patients have the same olfactory relief, psychophysically and subjectively, as those undergoing the trans-ethmoidal technique.

Key words: olfaction disorders, olfactory mucosa, sphenoid sinus, surgery

\section{Introduction}

Potential complications of endoscopic sinus surgery include impairment or loss of olfactory function. In particular, sphenoidotomy remains a challenging part of endoscopic sinus surgery with a potential for causing olfactory compromise due to its proximity to anatomical areas responsible for olfaction ${ }^{(1)}$. Specifically, olfactory epithelium overlies part of the nasal septum, cribriform plate, middle turbinate, and the superior turbinate.

Access to the sphenoid sinus can be surgically challenging.
Sphenoidotomy approaches have evolved and generally speaking, there are two methods to access the sphenoid sinus: either through the natural ostium (trans-sphenoethmoidal or via sphenoethmoidal recess) or by creating a second opening through the posterior ethmoids (trans-ethmoidal). Bolger et al. described the trans-ethmoidal approach in $2001^{(2)}$. However, differences in anatomy (attachment of the superior turbinate) may necessitate another approach. The trans-sphenoethmoidal technique is a described alternative ${ }^{(2)}$. The trans-sphenoethmoidal technique involves resection of the inferior one-third of the superior tur- 
binate. One of the concerns with this technique is the potential for disruption of olfactory epithelium in the superior turbinate through mechanisms such as direct or unrecognized damage, scarring, or oedema ${ }^{(2)}$.

However, whether or not the trans-sphenoethmoidal approach actually impacts olfactory function still remains unclear. In this study, we compared the effect on olfaction in both of these sphenoidotomy approaches.

\section{Materials and methods}

Patient population

A prospective cohort study was conducted at the St. Paul's Hospital Sinus Centre (SPHSC) in Vancouver, Canada with approval by the University of British Columbia Clinical Research Ethics Board. Individuals eligible for enrollment were diagnosed with CRS based on the Canadian clinical practice guidelines for sinusitis ${ }^{(4)}$. These individuals had persistent symptoms for at least 12 weeks with two or more clinical symptoms, which included facial congestion, facial pain, nasal obstruction, nasal discharge and a decreased sense of smell, as well as one objective finding on endoscopy or CT scan. Consecutive patients with bilateral sinus disease requiring bilateral maxillary antrostomy, complete ethmoidectomy, frontal sinustomy and sphenoidotomy were approached to partake in the study. All patients were consented to partake in the study between September 2010 and February 2012. Patients with tumours, allergic fungal rhinosinusitis, or polyps in the olfactory cleft/sphenoethmoidal recess were excluded from the study.

\section{Psychophysical olfactory measurement}

Patients had their smell tested psychophysically using the "Sniffin' Sticks" test. The "Sniffin' Sticks" test is a validated method for measuring olfactory function using odour-dispensing felt pens to test patients' olfactory threshold, their ability to discriminate between odours and their ability to identify specific odours. A composite numeric TDI (threshold, discrimination, and identification) score out of 48 is generated. Patients with a TDI score of 30 or above are considered normosmic, a score of 16 to 29 are considered hyposmic, and a score of 15 or below are considered anosmic ${ }^{(5,6)}$. Psychophysical measures were done pre-operatively and at 5 weeks post-operatively.

\section{Subjective olfactory measurement}

Visual analogue scores (VAS) on a $10 \mathrm{~cm}$ scale were done preoperatively and at 5 weeks post-operatively.

\section{Surgical approach}

A CT grading system created by Gheriani et al. ${ }^{(7)}$ and validated by Sunkaraneni et al. ${ }^{(8)}$ to assess the attachment of the superior turbinate to the sphenoid face determines the safest approach in performing a sphenoidotomy. The sphenoid face is divided into thirds at the level of the natural sphenoid ostium. The trans-ethmoidal approach was performed when the superior turbinate is attached to the sphenoid face within the medial one-third (type A) or middle one-third (type B). The transsphenoethmoidal approach was performed when the superior turbinate is attached to the lateral one-third (type $C$ ) or directly to the orbit (type D). Sunkaraneni's study simplified type A and type $B$ superior turbinate attachment as type I and type $C$ and type $D$ as type II ${ }^{(8)}$. If a patient required a trans-sphenoethmoidal approach based on the CT scan to at least one of the sphenoid sinuses, this was then performed on the opposite side as well. Since patients' treatment arm was dependent on the anatomy, patients were recruited until the minimum sample population per treatment arm was obtained. Post-operatively, all patients only used high-volume saline irrigation until their 5-week visit.

\section{Statistics}

A sample size calculation was completed prior to enrollment, which indicated a total of 48 subjects would be required to detect a significant difference between the sphenoidotomy approaches. The effect size for this calculation was designated as 3 points (6.25\%) between the pre and postoperative total TDI score, with type 1 probability of $5 \%(\alpha=0.05)$ and a type 2 probability of $20 \%(\beta=0.20)$. Twenty-four patients were required per arm.

Baseline characteristics were recorded for each subject, which included age (years), sex, disease severity (Lund-Mackay CT score), eosinophilia, nasal polyposis and history of sinus surgery. Pre-operative olfaction assessment was completed for each subject (Threshold, Discrimination, Identification) prior to surgery and 5 weeks post-FESS. Subjective patient-reported sense of smell was evaluated using a Visual Analogue Scale prior to surgery and 5 weeks post-FESS. Subjects were stratified by olfaction performance (i.e. anosmia, hyposmia or normosmia) based on results prior to surgery. Operative details including time of surgery (seconds), estimated blood loss (milliliters) and requirement of nasal septal reconstruction were also recorded. Continuous explanatory variables (age, LM score, olfaction score, time of surgery, estimated blood loss) were summarized with mean and standard deviation. Categorical explanatory variables (sex, eosinophilia, nasal polyposis, previous sinus surgery, nasal septal reconstruction, preoperative olfaction status) were summarized by count and relative proportion. The primary outcome variables were considered continuous and defined as the change in total olfaction score (TDI) and change in patient-reported sense of smell, between baseline and 5 weeks post-FESS evaluation. The parametric student t-test and two-proportion test were used to compare continuous and categorical varia- 
Table 1. Baseline characteristics.

\begin{tabular}{|c|c|c|c|c|}
\hline Baseline characteristics & $\begin{array}{l}\text { Trans-ethmoid }(\mathbf{n}=24) \\
\text { Mean (SD) }\end{array}$ & $\begin{array}{l}\text { Trans-sphenoethmoid }(n=24) \\
\text { Mean (SD) }\end{array}$ & $95 \% \mathrm{Cl}$ & p-value \\
\hline Mean age (years) & $47.0(13.4)$ & $56.6(10.8)$ & $-16.6,-2.4$ & 0.01 \\
\hline \multicolumn{5}{|l|}{ Sex } \\
\hline Males & $10(42 \%)$ & $12(50 \%)$ & $-0.4,0.2$ & 0.77 \\
\hline Females & $14(58 \%)$ & $12(50 \%)$ & $-0.2,0.4$ & 0.77 \\
\hline Eosinophilia & $2(8 \%)$ & $3(13 \%)$ & $-0.3,0.2$ & 1.0 \\
\hline Nasal polyposis & $8(33 \%)$ & $7(29 \%)$ & $-0.3,0.3$ & 1.0 \\
\hline Primary surgery & $12(50 \%)$ & $13(54 \%)$ & $-0.4,0.3$ & 1.0 \\
\hline $\begin{array}{l}\text { Disease severity } \\
\text { (Lund-Mackay score, /24) }\end{array}$ & $15.8(4.7)$ & $14.2(6.7)$ & $-1.8,5.0$ & 0.35 \\
\hline \multicolumn{5}{|l|}{ Operative details } \\
\hline NSR & $10(42 \%)$ & $5(21 \%)$ & $-0.1,0.5$ & 0.21 \\
\hline Time of surgery (seconds) & $128(38.6)$ & $111(46)$ & $-8.8,42.1$ & 0.19 \\
\hline Estimated blood loss (ml) & $478(404.6)$ & $540(513)$ & $-339.3,215.6$ & 0.66 \\
\hline \multicolumn{5}{|l|}{ Mean preoperative olfaction score } \\
\hline Threshold & $3.8(3.1)$ & $3.0(2.8)$ & $-0.9,2.5$ & 0.35 \\
\hline Discrimination & $6.7(4.6)$ & $6.8(4.5)$ & $-2.8,2.5$ & 0.90 \\
\hline Identification & $7.3(5.3)$ & $8.6(5.3)$ & $-4.4,1.7$ & 0.39 \\
\hline Total (TDI) & $17.8(11.7)$ & $18.5(11.3)$ & $-7.3,6.0$ & 0.83 \\
\hline \multicolumn{5}{|l|}{ Olfaction performance } \\
\hline Anosmia (TDI $\leq 15)$ & $8(33 \%)$ & $8(33 \%)$ & $-0.3,0.3$ & 1 \\
\hline Hyposmia (TDI $\geq 16, \leq 29$ ) & $13(54 \%)$ & $14(58 \%)$ & $-0.4,0.3$ & 1 \\
\hline Normosmia (TDI $\geq 30$ ) & $3(13 \%)$ & $2(8 \%)$ & $-0.2,0.3$ & 1 \\
\hline Patient-reported sense of smell & $3.7(3.2)$ & $3.0(2.6)$ & $-1.0,2.4$ & 0.41 \\
\hline
\end{tabular}

Table 2. Comparison of change in olfaction performance and patient-reported sense of smell between baseline and 5 weeks.

\begin{tabular}{|c|c|c|c|c|}
\hline $\begin{array}{c}\text { Difference pre- vs. postoperative } \\
\text { performance }\end{array}$ & $\begin{array}{l}\text { Trans-ethmoid }(n=24) \\
\text { Mean (SD) }\end{array}$ & $\begin{array}{l}\text { Trans-sphenoethmoid }(n=24) \\
\text { Mean (SD) }\end{array}$ & $95 \% \mathrm{Cl}$ & p-value \\
\hline \multicolumn{5}{|l|}{ Olfaction score } \\
\hline Threshold & $0.1(1.5)$ & $0.5(3.1)$ & $-1.9,1.0$ & 0.54 \\
\hline Discrimination & $0.3(2.7)$ & $1.25(4.8)$ & $-3.2,1.3$ & 0.40 \\
\hline Identification & $1.9(4.2)$ & $0.4(4.2)$ & $-0.9,4.0$ & 0.21 \\
\hline Total & $2.3(6.8)$ & $1.7(9.3)$ & $-4.2,5.3$ & 0.81 \\
\hline Patient-reported sense of smell & $0.4(3.1)$ & $1.7(2.8)$ & $-3.0,0.4$ & 0.13 \\
\hline
\end{tabular}

Table 3. Comparison of unadjusted and adjusted regression estimates from multivariate linear regression model accounting for age.

*The reference group was patients receiving the Trans-ethmoid approach.

\begin{tabular}{lcccc}
\multicolumn{1}{c}{ Variables } & Unadjusted estimate (SE) & p-value & Adjusted estimate (SE) & p-value \\
\hline $\begin{array}{l}\text { Change in olfaction (TDI score) } \\
\begin{array}{l}\text { Change in patient-reported sense of } \\
\text { smell (VAS score) }\end{array}\end{array}$ - $^{1.31(0.86(1.67)}$ & 0.18 & $0.16(2.55)$ & 0.95 \\
\hline
\end{tabular}


bles for significant differences respectively. Probability values less than 0.05 was considered significant. Multivariate linear regression was used to model the relationship between the change in olfaction performance before and 5 weeks post-FESS and sphenoidotomy procedure. Significant baseline variables were added to the model to obtain adjusted effects to evaluate potential confounding. Statistical analysis was completed using GraphPad Prism Version 5.0a (GraphPad Software, Inc., 2008) and RStudio Version 0.95.265 (RStudio, Inc., 2011).

\section{Results}

A total of 48 adult CRS patients were enrolled into this study, with equal subjects $(n=24)$ in the TE and TSE groups. Baseline characteristics are summarized in Table 1. Mean age was significantly different in the TE (47.0 years) and TSE (56.6 years) groups respectively $(p=0.01,95 \% \mathrm{Cl}:-16.6,-2.4)$. The distribution of sex, nasal polyposis, eosinophilia and previous sinus surgery were comparable (Table 1). Preoperative disease severity was not significantly different in both treatment groups (15.8 TE vs. 14.2 TSE, $p=0.35,95 \% \mathrm{Cl}:-1.8,5.0$ ). Preoperative olfaction performance was comparable between the TE and TSE groups for total TDI score (17.8 vs. $18.5, \mathrm{p}=0.83,95 \% \mathrm{Cl}:-7.3,6.0)$. Performance in Threshold, Discrimination and Identification are summarized in Table 1. Similarly, preoperative patient-reported sense of smell was not significantly between TE and TSE groups, respectively (3.7 vs. 3.0, $\mathrm{p}=0.41,95 \% \mathrm{Cl}:-1.0,2.4$ ).

Olfaction performance and perception was re-evaluated 5 weeks after surgery and compared to preoperative results (Figure 1). Overall TDI score and sense of smell improved for the TE (TDI: $2.3 \pm 6.8$, VAS: $0.4 \pm 3.1$ ) and TSE (TDI: $1.7 \pm 9.3$, VAS: $1.7 \pm 2.8$ ) groups (Table 2 ). These findings were not statistically

Table 4. Stratification by preoperative olfaction performance.

\begin{tabular}{|c|c|c|}
\hline Anosmic $(n=16)$ & $\begin{array}{c}\text { Trans-ethmoid }(\mathbf{n}=\mathbf{8}) \\
\text { Mean }(\mathrm{SD} / \%)\end{array}$ & $\begin{array}{l}\text { Trans-sphenoethmoid }(\mathbf{n}=\mathbf{8}) \\
\text { Mean }(\mathrm{SD} / \%)\end{array}$ \\
\hline Age (years) & $49(13)$ & $55(9.5)$ \\
\hline Males & $1(13 \%)$ & $2(25 \%)$ \\
\hline Eosinophilia & $2(25 \%)$ & $1(13 \%)$ \\
\hline Nasal Polyposis & $6(75 \%)$ & $4(50 \%)$ \\
\hline Primary surgery & $3(38 \%)$ & $5(63 \%)$ \\
\hline Bilateral disease severity (LM score) & $19.0(6.3)$ & $16.1(8.2)$ \\
\hline Nasal Septal Reconstruction & $1(13 \%)$ & $2(25 \%)$ \\
\hline \multicolumn{3}{|l|}{ Change in Olfaction (Pre vs. Post) } \\
\hline Total TDI & $3.8(9.6)$ & $6.6(9.8)$ \\
\hline Patient-reported sense of smell & $1.3(2.5)$ & $1.7(2.7)$ \\
\hline Hyposmic ( $n=27)$ & $\begin{array}{l}\text { Trans-ethmoid }(\mathbf{n}=13) \\
\text { Mean }(\mathrm{SD} / \%)\end{array}$ & $\begin{array}{c}\text { Trans-sphenoethmoid }(n=14) \\
\text { Mean (SD/\%) }\end{array}$ \\
\hline Age & 47 (14) & $58(11)$ \\
\hline Males & $8(62 \%)$ & $9(64 \%)$ \\
\hline Eosinophilia & 0 & $1(7 \%)$ \\
\hline Nasal Polyposis & $2(15 \%)$ & $3(21 \%)$ \\
\hline Primary surgery & $7(54 \%)$ & $6(43 \%)$ \\
\hline Bilateral disease severity & $13.6(3.9)$ & $12.9(6.0)$ \\
\hline Nasal Septal Reconstruction & $6(46 \%)$ & $2(14 \%)$ \\
\hline \multicolumn{3}{|l|}{ Change in Olfaction Score (Pre vs. Post) } \\
\hline Total TDI & $2.4(5.0)$ & $-0.1(8.7)$ \\
\hline Patient-reported sense of smell & $0.2(2.5)$ & $2.0(2.9)$ \\
\hline Normosmic $(n=5)$ & $\begin{array}{c}\text { Trans-ethmoid }(\mathbf{n}=\mathbf{3}) \\
\text { Mean }(\mathrm{SD} / \%)\end{array}$ & $\begin{array}{l}\text { Trans-sphenoethmoid }(\mathbf{n}=\mathbf{2}) \\
\text { Mean }(\mathrm{SD} / \%)\end{array}$ \\
\hline Age & $45(1)$ & $46(14.8)$ \\
\hline Males & $1(33 \%)$ & $1(50 \%)$ \\
\hline Eosinophilia & 0 & $1(50 \%)$ \\
\hline Nasal Polyposis & 0 & 0 \\
\hline Primary surgery & $2(66 \%)$ & $2(100 \%)$ \\
\hline Bilateral disease severity & $16.3(6.5)$ & $15.0(7.1)$ \\
\hline Nasal Septal Reconstruction & $3(100 \%)$ & $1(50 \%)$ \\
\hline \multicolumn{3}{|l|}{ Change in Olfaction Score (Pre vs. Post) } \\
\hline Total TDI & $-2.3(4.0)$ & $-5.5(2.1)$ \\
\hline Patient-reported sense of smell & $-1.1(6.8)$ & $-0.3(3.9)$ \\
\hline
\end{tabular}




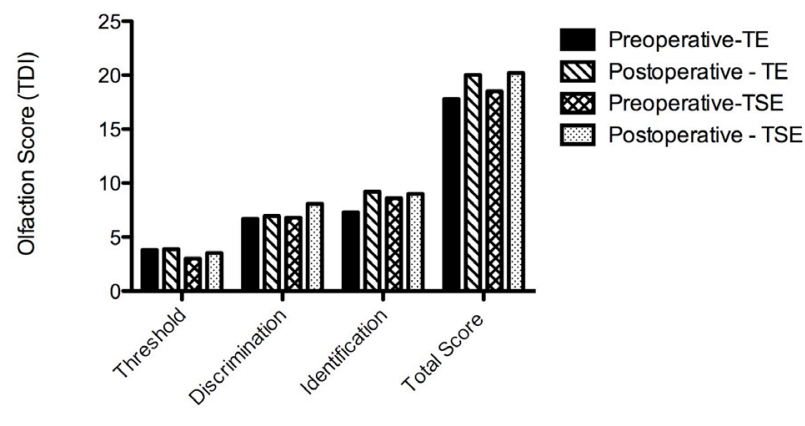

Figure 1. Comparison of mean pre- and postoperative olfaction score.

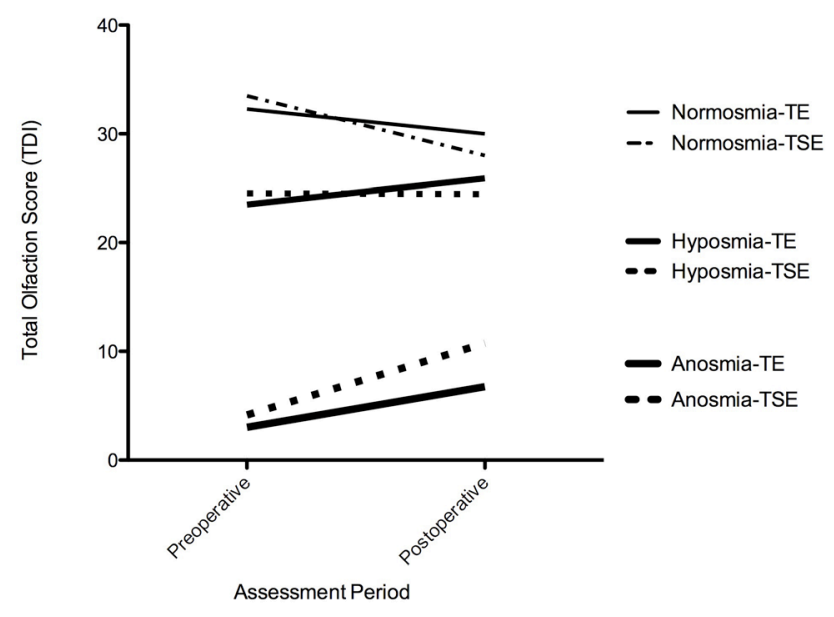

Figure 2. Change in olfaction score for Trans-ethmoid (TE) and Transsphenoethmoid (TSE) stratified by preoperative olfaction performance category.

significant ( $p=0.81,95 \% \mathrm{Cl}:-4.2,5.3)$. Comparing the TE and TSE groups, non-significant differences were found in Threshold ( $p$ $=0.54,95 \% \mathrm{Cl}:-1.9,1.0)$, Discrimination ( $p=0.40,95 \% \mathrm{Cl}:-3.2$, 1.3 ) and Identification ( $p=0.21,95 \% \mathrm{Cl}:-0.9,4.0)$ performance categories. On average, the difference in patient-reported sense of smell between pre- and postoperative evaluation did not significantly differ between the study groups $(p=0.13,95 \% \mathrm{Cl}$ : -3.0 , $0.4)$. A non-significant association was found in the difference of olfaction score $(p=0.95)$ and patient-reported sense of smell $(p=0.10)$ before and 5 weeks post-FESS between sphenoid approaches, when adjusting for age (Table 3 ).

Patients were stratified by olfaction performance prior to surgery. Overall, 16 (34\%) presented with anosmia, 27 (56\%) were hyposmic and 5 (10\%) were normosmic prior to surgery and were similarly distributed between the TE and TSE groups (Table 4). Anosmic patients were found to improve in total olfaction score by $3.8 \pm 9.6$ points in the TE and $6.6 \pm 9.8$ points in the TSE groups. This trend was similar in hyposmic patients receiving the TE approach $(2.4 \pm 5.0)$ but not those in the TSE group (-0.1 $\pm 8.7)$. Interestingly, normosmic patients were found to have reduced olfaction performance in both the TE $(-2.3 \pm 4.0)$ and TSE $(-5.5 \pm 2.1)$ groups. Patients stratificed by preoperative olfaction category, comparing change in olfaction score are displayed in Figure 2.

\section{Discussion}

There is general consensus following sinus surgery that patients' olfactory function improves. There are a number of short-term studies, ranging from weeks to a few months, as well as a few long-term studies illustrating sinus surgery's effectiveness in treating olfactory dysfunction ${ }^{(9-12)}$. The percentage of improvement post-functional endoscopic sinus surgery varies between studies ranging from 13 to $91 \%{ }^{(13)}$. Schriever et al. ${ }^{(12)}$ showed $18.6 \%$ of patients had improved their smell one year after surgery and only $1 \%$ of patients indicated a decline in their smell. Their study also used the same psychophysical olfactory measure, "Sniffin' Sticks". Delank et al.'s ${ }^{(14)}$ short-term study illustrated that $8 \%$ of patients post-sinus surgery had a loss in smell at their two-month follow-up. It is hypothesized that one of the reasons patients complain of olfactory loss may be secondary to loss of olfactory mucosa due to surgical technique.

It is important to spare as much olfactory mucosa when performing sinus surgery. The amount of olfactory mucosa changes as we age. A fetus has olfactory mucosa lining the roof of the nasal cavity down to the mid-portion of the nasal septum and onto the superior turbinate; however, as we age, the olfactory mucosa becomes replaced by respiratory epithelium leading to patchy olfactory mucosa ${ }^{(15,16)}$. As for the amount of olfactory epithelium extending down to the inferior aspect of the turbinate, it is unclear how much is actually present. Lane et al. ${ }^{(1)}$ removed one-third of the inferior aspect of the superior turbinate of four healthy patients and found olfactory nerves present in all the samples, whereas Say et al. ${ }^{\left({ }^{17)}\right.}$ assessed 55 samples from the inferior one-third of superior turbinates and found olfactory neuroepithelium in one-sixth of the patients. Moreover, to our knowledge, Say's research is the only study that evaluated olfactory function of patients who had their superior turbinates partially resected for access to the natural sphenoid ostium. Our study supports their finding that it is rare to have a clinically significant amount of olfactory mucosa within the bottom third of the superior turbinate, given that our patients showed no difference in olfaction whether they had the TSE or TE approach.

Our approach in entering the sphenoid depends on the position of the superior turbinate attachment to the sphenoid face (Parson's ridge). We use a CT grading system created by Gheriani et al. to assess the attachment of the superior turbinate ${ }^{(7)}$ and validated by Sunkaraneni et al. ${ }^{(8)}$. We favor the trans-spheno- 
ethmoidal when the superior turbinate attached to the lateral one-third of the sphenoid face or lateral (type I). This decreases the chances of entering the orbit, optic nerve or internal carotid artery. Otherwise, we prefer the trans-ethmoidal approach because the trans-sphenoethmoidal approach not only has hypothetical risks of olfactory dysfunction but there is also the possibility of iatrogenic cerebrospinal fluid (CSF) leak. The transsphenoethmoidal approach can be done in a systematic way; it is important to use a sharp cutting instrument so that twisting of the superior turbinate is avoided, which could lead to out fracturing of the entire superior turbinate or CSF leak.

The finding of decreased olfaction in both the TSE and TE groups who were normosmic raises a few pertinent implications. Generally, our study supports the idea that patients with compromised olfactory function will experience some improvement in olfactory capacity following sinus surgery, regardless of whether a TSE or TE approach is used. However, patients with uncompromised olfactory function are intuitively unable to improve beyond normal. This emphasizes the need to counsel normosmic patients preoperatively on the possibility of a decrease in their olfactory capacity. Additionally, this finding demonstrates the importance of stratifying patients and subgroup analysis in olfactory studies, especially since these subgroups have important clinical differences.

Our study demonstrates that there is no difference in olfaction following the two sphenoidotomy approaches. It is important to note that we grouped patients according to anatomical attachment of the superior turbinate, as opposed to randomized grouping. Therefore, care should be taken in interpreting our results. However, we would suggest that this is a more practical comparison, given that the main priority of entry into the sphenoid sinus is avoidance of major surgical complications (e.g. carotid injury), with limitation of olfactory compromise being a secondary priority.

There are a few other limitations with this study. This is a short-term study that shows no difference between the two techniques but long-term studies may elucidate a difference between these two surgical approaches. Limiting the amount of resection leads to less scarring and iatrogenic inflammatory changes, which may provide to be beneficial for those undergoing the trans-ethmoidal approach. A long-term study with correlation to endoscopic scores, such as the Javer-Philpott endoscopic grading scale, which looks at the each sinus cavity individually as well as the olfactory cleft will provide insight into how the each approaches differ over time ${ }^{(18)}$. Our study did not account for the potential impact of chronic sphenoid disease on the olfactory mucosa along the superior turbinate. Chronic infection of the nasal mucosa changes olfactory epithelium into respiratory epithelium ${ }^{(3)}$. Given that Lane's study found olfactory nerves within the inferior portion of the superior turbinate of healthy patients, one could hypothesize a much larger portion of the superior turbinate is void of olfactory mucosa in patients with chronic sphenoid disease. Nonetheless, we strongly favor minimal removal of the superior turbinate if one needs access to the natural sphenoid ostia. Studies have also shown that nasal polyposis and eosinophilia are predictive factors for improved olfaction following nasal surgery ${ }^{(12)}$. Nasal polyposis was not controlled in this study except for patients were excluded for having nasal polyps within the olfactory cleft/sphenoethmoidal recess. During the study design conception, it was felt that if the areas that contained olfactory mucosa were non-diseased and did not require debridement, then the degree of nasal polyps in the rest of the nasal cavity played a minimal role with olfaction. However, in retrospect, this study may have improved if these predictive factors, degree of nasal polyps within the entire nasal cavity and eosinophilia, were controlled.

\section{Conclusion}

In a specifically selected group of patients, the TSE sphenoidotomy approach does not carry an increased risk of olfactory compromise compared to the TE sphenoidotomy approach. Regardless, the TSE approach should be done meticulously to minimize a theoretical possibility of olfactory loss not identified in our study. Further long-term, prospective studies are needed to better characterize the differences in the TE as TSE approaches.

\section{Acknowledgements}

This study received no funding. We are thankful to Rachelle Dar Santos for assistance with institutional ethics review board application.

\section{Authorship contribution}

AT, BAC: study design, data analysis, manuscript preparation ARH, JM: data collection, data analysis

$\mathrm{IH}, \mathrm{VS}, \mathrm{AJ}$ : study design, manuscript review

\section{Conflict of interest}

No conflicts of interest to disclose.

\section{References}

1. Lane AP, Gomez G, Dankulich T, Wang H Bolger WE, Rawson NE. The superior turbinate as a source of functional human olfac- tory receptor neurons. Laryngoscope. 2002 112: 1183-1189.

2. Orlandi RR, Lanza DC, Bolger WE, Clerico DM, Kennedy DW. The forgotten turbinate: the role of the superior turbinate in endoscopic sinus surgery. Am J Rhinol. 1999; 13: 251-259.

3. Kern RC. Chronic sinusitis and anosmia: 
pathologic changes in the olfactory mucosa. Laryngoscope. 2000; 110: 1071-1077.

4. Desrosiers M, Evans GA, Keith PK, Wright ED, Kaplan A, Bouchard J, et al. Canadian clinical practice guidelines for acute and chronic rhinosinusitis. J Otolaryngol Head Neck Surg. 2011; 40 Suppl 2: S99-193.

5. Hummel T, Kobal G, Gudziol H, Mackay-Sim A. Normative data for the "Sniffin' Sticks" including tests of odor identification, odor discrimination, and olfactory thresholds: an upgrade based on a group of more than 3,000 subjects. Eur Arch Otorhinolaryngol. 2007; 264: 237-243.

6. Hummel $T$, Sekinger $B$, Wolf $S R$, Pauli $E$ Kobal G. 'Sniffin' sticks': olfactory performance assessed by the combined testing of odor identification, odor discrimination and olfactory threshold. Chem Senses. 1997; 22: 39-52.

7. Gheriani H, Flamer D, Orton T, Mechor B, Javer AR. A comparison of two sphenoidotomy approaches using a novel computerized tomography grading system. Am J Rhinol Allergy. 2009; 23: 212-217.

8. Sunkaraneni VS, Qian $H$, Wong $H$, Javer A. Validation of a grading system for the attachment of the superior turbinate to the sphenoid face. Int Forum Allergy Rhinol. 2012; 2: 411-414.

9. Konstantinidis I, Triaridis S, Printza A, Vital
V, Ferekidis E, Constantinidis J. Olfactory dysfunction in nasal polyposis: correlation with computed tomography findings. ORL J Otorhinolaryngol Relat Spec. 2007; 69: 226-232.

10. Konstantinidis I, Witt M, Kaidoglou K, Constantinidis J, Gudziol V. Olfactory mucosa in nasal polyposis: implications for FESS outcome. Rhinology. 2010; 48: 47-53.

11. Litvack JR, Mace J, Smith TL. Does olfactory function improve after endoscopic sinus surgery? Otolaryngol Head Neck Surg. 2009; 140: 312-319.

12. Schriever VA, Gupta N, Pade J, Szewczynska $M$, Hummel T. Olfactory function following nasal surgery: a 1-year follow-up. Eur Arch Otorhinolaryngol. 2013; 270: 107-111.

13. Bonfils P, Malinvaud D, Soudry Y, Devars du Maine $M$, Laccourreye $O$. Surgical therapy and olfactory function. B-Ent. 2009; 5 Suppl 13: 77-87.

14. Delank KW, Stoll W. Olfactory function after functional endoscopic sinus surgery for chronic sinusitis. Rhinology. 1998; 36: 15-19.

15. Nakashima T, Kimmelman CP, Snow JB, Jr. Structure of human fetal and adult olfactory neuroepithelium. Arch Otolaryngol. 1984; 110: 641-646.

16. Paik SI, Lehman MN, Seiden AM, Duncan HJ, Smith DV. Human olfactory biopsy. The influence of age and receptor distribution.
Arch Otolaryngol Head Neck Surg. 1992; 118: 731-738.

17. Say P, Leopold D, Cochran G, Smith L, Greiner T. Resection of the inferior superior turbinate: does it affect olfactory ability or contain olfactory neuronal tissue? Am J Rhinol. 2004; 18: 157-160.

18. Philpott CM, Javer AR, Clark A. Allergic fungal rhinosinusitis - a new staging system. Rhinology. 2011; 49: 318-323.

Andrew Thamboo

St. Paul's Sinus Centre

1081 Burrard Street

Vancouver, BC

V6Z 1 Y6 Canada

Tel: +1-604-806-9926

Fax: +1-604-806-8447

E-mail: andrew.thamboo@gmail.com 